\title{
Discovery of a variable star population in NGC 2808 (Corrigendum)
}

\author{
T. Michael Corwin ${ }^{1, \star}$, M. Catelan ${ }^{2, \star}$, J. Borissova ${ }^{2, \star}$, and H. A. Smith ${ }^{3}$ \\ 1 Department of Physics, University of North Carolina at Charlotte, Charlotte, NC 28223, USA \\ e-mail: mcorwin@uncc.edu \\ 2 Pontificia Universidad Católica de Chile, Departamento de Astronomía y Astrofísica, Av. Vicuña Mackenna 4860, $782-0436$ Macul, \\ Santiago, Chile \\ e-mail: [mcatelan;jborisso]@astro.puc.cl \\ 3 Dept. of Physics and Astronomy, Michigan State University, East Lansing, MI 48824, USA \\ e-mail: smith@pa.msu.edu
}

\section{A\&A 421, 667-672 (2004), DOI: 10.1051/0004-6361:20035944}

Key words. stars: horizontal-branch - globular clusters: individual: NGC 2808 - stars: variables: RR Lyr - errata, addenda

It has recently been brought to our attention that the coordinates for some of the NGC 2808 variable stars, as provided in Table 1 of our paper, were incorrect. A revised table with the corrected coordinates is given below. The $(x, y)$ coordinates were also recomputed, and are in units of arcseconds relative to the cluster center, estimated to lie at RA $=09: 12: 02.74, \mathrm{Dec}=-64: 51: 53.7$ (J2000.0).

In the process of revising these coordinates, it was found that, very close to our V20, and matching closely the position given for V20 in our finding chart, another previously unidentified variable star is present, which is likely a BL Her (type II Cepheid) with a period of about $2.1 \mathrm{~d}$. This discovery will be reported on more fully in Kunder et al. (in prep.), but the star is also included in Table 1, for the sake of completeness.

We warmly thank C. M. Clement for calling our attention to the problem with our original coordinates, and also P. B. Stetson, A. Kunder, and P. Amigo, for their help in thoroughly revising the list of coordinates, and for reporting on the discovery of V51.

Table 1. Variable stars detected in NGC 2808.

\begin{tabular}{lccccccc}
\hline \hline Name & $x$ & $y$ & RA & Dec $($ J2000 $)$ & Period $(\mathrm{d})$ & Type & Comment \\
\hline V1 & 111.4 & -26.6 & $9: 12: 20.22$ & $-64: 52: 20.5$ & & SR & \\
V6 & 174.2 & -284.0 & $9: 12: 30.08$ & $-64: 56: 37.9$ & 0.53897 & RRab & \\
V10 & -37.3 & -89.5 & $9: 11: 56.86$ & $-64: 53: 23.2$ & 1.76528 & Cepheid & \\
V12 & -42.2 & 104.0 & $9: 11: 56.15$ & $-64: 50: 09.6$ & 0.30578 & RRc & \\
V13 & 131.4 & -316.7 & $9: 12: 23.34$ & $-64: 57: 10.6$ & 0.21 & RRc & RRe? W UMa? \\
V14 & 67.8 & 49.9 & $9: 12: 13.40$ & $-64: 51: 04.0$ & 0.60 & RRab & \\
V15 & 50.0 & 38.1 & $9: 12: 10.60$ & $-64: 51: 15.7$ & 0.61 & RRab & \\
V16 & 40.7 & -57.7 & $9: 12: 09.11$ & $-64: 52: 51.5$ & 0.59 & RRab & \\
V17 & 27.2 & -3.4 & $9: 12: 07.01$ & $-64: 51: 57.2$ & 0.38 & RRc & \\
V18 & 24.4 & -23.2 & $9: 12: 06.57$ & $-64: 52: 17.0$ & 0.58 & RRab & not phased in $V$ \\
V19 & 22.4 & -16.8 & $9: 12: 06.25$ & $-64: 52: 10.6$ & 0.51 & RRab & \\
V20 & 16.4 & 27.3 & $9: 12: 05.32$ & $-64: 51: 26.5$ & 0.29 & RRc & \\
V21 & 7.2 & 27.8 & $9: 12: 03.88$ & $-64: 51: 26.0$ & 0.60 & RRab & \\
V22 & 7.4 & -1.8 & $9: 12: 03.90$ & $-64: 51: 55.6$ & 0.54 & RRab & large scatter in $V$ \\
V23 & 3.3 & -26.0 & $9: 12: 03.25$ & $-64: 52: 19.7$ & 0.27 & RRc & \\
V24 & -0.7 & -17.0 & $9: 12: 02.63$ & $-64: 52: 10.7$ & 0.27 & RRc & Blazhko? \\
V25 & -25.9 & 25.9 & $9: 11: 58.68$ & $-64: 51: 27.8$ & 0.49 & RRab & \\
V26 & -61.6 & 22.9 & $9: 11: 53.08$ & $-64: 51: 30.7$ & 0.37 & RRc & \\
V27 & -57.9 & -66.1 & $9: 11: 53.64$ & $-64: 52: 59.7$ & 0.57 & RRab & \\
V28 & -77.6 & 25.7 & $9: 11: 50.58$ & $-64: 51: 27.8$ & 0.28 & RRc & \\
V29 & -14.2 & 14.6 & $9: 12: 00.52$ & $-64: 51: 39.1$ & 1.97 & Cepheid? & \\
V30 & -85.3 & 0.9 & $9: 11: 49.36$ & $-64: 51: 52.7$ & 1.47 & EB & $\beta$ Lyr? \\
V51 & 17.7 & 22.7 & $9: 12: 05.52$ & $-64: 51: 31.1$ & 2.11 & BL Her & \\
\hline
\end{tabular}

\footnotetext{
^ Visiting Astronomer, European Southern Observatory, La Silla,
} Chile. 